\title{
Hybrid Based Resource Provisioning in Cloud
}

\author{
N.Karthika \\ Vivekanandha College of \\ Engineering For Women \\ Tiruchengode, India
}

\author{
K.Prabhakar \\ Vivekanandha College of \\ Engineering For Women \\ Tiruchengode, India
}

\author{
R.Sangeetha \\ Vivekanandha College of \\ Engineering For Women \\ Tiruchengode, India
}

\begin{abstract}
The data centres and energy consumption characteristics of the various machines are often noted with different capacities. The public cloud workloads of different priorities and performance requirements of various applications when analysed we had noted some invariant reports about cloud. The Cloud data centres become capable of sensing an opportunity to present a different program. In out proposed work, we are using a hybrid method for resource provisioning in data centres. This method is used to allocate the resources at the working conditions and also for the energy stored in the power consumptions. Proposed method is used to allocate the process behind the cloud storage.
\end{abstract}

Keywords: Cloud workload, Hybrid resource provisioning, Cloud storage and Invariant reports.

\section{INTRODUCTION}

Cloud Computing is the common buzzword in today's Information Technology. Cloud computing platforms are rapidly emerging as the preferred option for hosting applications in many business contexts [5]. An important feature of the cloud that differentiates it from traditional services is its apparently infinite amount of resource capacity (e.g. CPU, storage, Network) offered at a competitive rate. It eliminates the need for setting up infrastructure which takes several months. Start-up Companies need not invest on the infrastructure because the resources are available in the cloud [6]. Cloud Computing enables users to acquire resources dynamically and elastically.

A major challenge in resource provisioning technique is to determine the right amount of resources required for the execution of work in order to minimize the financial cost from the perspective of users and to maximize the resource utilization from the perspective of service providers [4]. So, Cloud computing is one of the preferred options in today's enterprise. Resource provisioning means the selection, deployment, and run-time management of software (e.g., database management servers, load balancers) and hardware resources (e.g., CPU, storage, and network) for ensuring guaranteed performance for applications. This resource provisioning takes Service Level Agreement (SLA) into consideration for providing service to the cloud users. This is an initial agreement between the cloud users and cloud service providers which ensures Quality of Service (QoS) parameters like performance, availability, reliability, response time etc.

Based on the application needs Static Provisioning/Dynamic Provisioning and Static/Dynamic Allocation of resources have to be made in order to efficiently make use of the resources without violating SLA and meeting these QoS parameters. Over provisioning and under provisioning of resources must be avoided. Another important constraint is power consumption. Care should be taken to reduce power consumption, power dissipation and also on VM placement.
There should be techniques to avoid excess power consumption. So the ultimate goal of the cloud user is to minimize cost by renting the resources and from the cloud service provider's perspective to maximize profit by efficiently allocating the resources. In order to achieve the goal the cloud user has to request cloud service provider to make a provision for the resources either statically or dynamically so that the cloud service provider will know how many instances of the resources and what resources are required for a particular application. By provisioning the resources, the QoS parameters like availability, throughput, security, response time, reliability, performance etc must be achieved without violating SLA.

Platform as a Service is a way to rent hardware, operating systems, storage and network capacity over the internet. It delivers a computing platform or software stack as a service to run applications. This can broadly be defined as application development environment offered as a 'service' by the vendors. The development community can use these platforms to code their applications and then deploy the applications on the infrastructure provided by the cloud vendor. Here again, the responsibility of hosting and managing the required infrastructure will be with the cloud vendor. AppEngine, Bungee Connect, LongJump, Force.com, WaveMaker are all instances of PaaS.

\section{RELATED WORKS}

From the last fewer, cloud computing has evolved as delivering software and hardware services over the internet. The extensive research is going on to extend the capabilities of cloud computing. Given below present related work in the area of cloud's scalability and resource provisioning in cloud computing.

In 2010 ChunyeGong, Jie Liu, Oiang Zhang, Haitao Chen and Zhenghu has discussed Characteristics of Cloud Computing. In this paper summarize the general characteristics of cloud computing which will help the development and adoption of this rapidly evolving technology. The key characteristics of cloud computing

are low cost, high reliability, high scalability, security. To make clear and essential of cloud computing, proposes the 
characteristics of this area which make the cloud computing being cloud computing and distinguish it from other research area. The cloud computing has its own technical, economic, user experience characteristics. The service oriented, loose coupling, strong fault tolerant, business model and ease use are main characteristics of cloud computing. Abstraction and accessibility are two keys to achieve the service oriented conception. In loose coupling cloud computing run in a clientserver model. The client or cloud users connect loosely with server or cloud providers. Strong fault tolerant stand for main technical characteristics. The ease use user experience characteristic helps cloud computing being widely accepted by non computer experts. These characteristics expose the essential of cloud computing. [1]

In 2010 Pushpendra kumar pateria, Neha Marria discussed resource provisioning in sky environment. Resource manager is used for resource provisioning and allocate of resource as user request. Offer the rule based resource manager in sky environment for utilization the private cloud resource and security requirement of resource of critical application and data .Decision is made on the basis of rule. Performance of resource manager is also evaluated by using cloudsim on basis of resource utilization and cost in sky environment. Set priorities request and allocate resource accordingly. Sky computing provides computing concurrent access to multiple clouds according user requirement. Define the Cloud services like Software as a service (SaaS), Platform as a Service $(\mathrm{PaaS})$ and Infrastructure as a service. [2]

In 2010 Zhang Yu Hua, Zhang Jian ,Zhang Wei Hua present argumentation about the intelligent cloud computing system and Data warehouse that record the inside and outside data of Cloud Computing System for data analysis and data mining. Management problem of CCS are: balance between capacity and demand, capacity development planning, performance optimization, system safety management. Architecture of the Intelligence cloud computing system is defined with Data source, data warehouse and Cloud computing management information system. [3]

In 2008 discussed about the Phoenix by Jianfeng Zhan, Lei Wang, Bipo Tu, Yong Li, Peng Wang, Wei Zhou and Dan Meng. In this paper discuss the designed and implemented cloud management system software Phoenix Cloud. Different department of large organization often maintain dedicate cluster system for different computing loads. The department from big organization have operated cluster system with independent administration staffs and found many problem like resource utilization rates of cluster system are varying, dedicated cluster systems cannot provision enough resources and number of administration staff for cluster system is high. So here designed and implemented cloud management system software Phoenix Cloud to consolidate high performance computing jobs and Web service application on shared cluster system. Phoenix Cloud decreases the scale of required cluster system for a large organization.

improves the benefit of scientific computing department, and provisions resources. [4]

In 2010 Shu-Ching Wang, Kuo-Qin Yan, Wen-Pin Liao and Shun-Sheng Wang discussed about Load Balancing in ThreeLevel Cloud Computing Network. Cloud computing utilize low power host to achieve high reliability. In this Cloud computing is to utilize the computing resources on the network to facilitate the execution of complicated tasks that require large-scale computation. Use the OLB scheduling algorithm is used to attempt each node keep busy and goal of load balance. Also proposed LBMM (Load Balance MinMin) scheduling algorithm can make the minimum execution time of each task on cloud computing environment and this will improve the load unbalance of the Min-Min. In order to reach load balance and decrease execution time for each node in the three-level cloud computing network, the OLB and LBMM scheduling algorithm are integrated. The load balancing of three-level cloud computing network is utilized all calculating result could be integrated first by the secondlevel node [5]

In January 31, 2011, Sivadon Chaisiri, Bu-Sung Lee, and Dusit Niyato discuss about the Optimization of Resource Provisioning Cost. Under the resource provisioning optimal cloud provisioning algorithm illustrates virtual machine management that consider multiple provisioning stages with demand price uncertainty. In this task system model of cloud computing environment has been thoroughly explained using various techniques such as cloud consumer, virtual machine and cloud broker in details. [8]

The agent-based adaptive resource allocation is discussed in 2011 by the Gihun Jung, Kwang Mong Sim. In this paper the provider needs to allocate each consumer request to an appropriate data center among the distributed data centers because these consumers can satisfy with the service in terms of fast allocation time and execution response time. Service provider offers their resources under the infrastructure as a service model. For IaaS the service provider delivers its resources at the request of consumers in the form of VMs. To find an appropriate data center for the consumer request, propose an adaptive resource allocation model considers both the geographical distance between the location of consumer and datacenters and the workload of data center. With experiment the adaptive resource allocation model shows higher performance. An agent based test bed designed and implemented to demonstrate the proposed adaptive resource allocation model. The test bed implemented using JAVA with JADE (Java Agent Development framework). [9]

\section{SYSTEM ARCHITECTURE}

Dynamically adjusting the number of machines has each type to minimize total energy consumption and performance penalty in terms of scheduling delay. In my proposed using the hybrid method for resource provisioning in data centers. This method is used to allocate the resources at the working conditions and also energy stored for the power consumptions. Proposed method is used to allocate the process behind the cloud storage.

\subsection{User Interface Design}

In this module we design the windows for the project. These windows are used to send a message from one to another. In this module mainly we are focusing the login design page with the Partial knowledge information. Application Users need to view the application they need to login through the User Interface GUI is the media to connect User and Media Database. 


\subsection{Dynamic Capacity Provisioning In Data Centers}

In this section we address the simulation of heterogeneous active machine. Here we are going to create difference machine for stored based on the client demand that is production data centers often comprise heterogeneous machines with different capacities and energy consumption characteristics..The energy level consumption is updated by the cloud service provider which belongs to the datacenter. Data center have more right the route the heterogeneous active machine. This area regulates Heterogeneous active machine creation. Also it regulates memory consumption and key challenge that often has been overlooked or considered difficult to address is heterogeneity,

\subsection{Machine Heterogeneity Model Approach}

This simulation addresses the production data centers often comprise several types of machines from multiple update. They have heterogeneous processor architectures and speeds, hardware features, memory and disk capacities. Consequently, they have different runtime energy consumption rates. Here we are going to create difference machine for stored based on the client demand that is production data centers often comprise heterogeneous machines with different capacities and energy consumption characteristics..The energy level consumption is updated by the cloud service provider which belongs to the datacenter.

\subsection{Resource Monitoring and Management System}

Production data centers receive a vast number of heterogeneous resource requests with diverse resource demands, durations, priorities and performance. The heterogeneous nature of both machine and workload in production cloud environments has profound implications on the design of DCP schemes. Here we address accurate characterization of both workload and machine heterogeneities. Standard K-means clustering, we show that the heterogeneous workload can be divided into multiple task classes with similar characteristics in terms of resource and performance objectives.

\subsection{Dynamic Capacity Provisioning Approach}

The workload traces contain scheduling events, resource demand and usage records. The job is an application that consists of one or more tasks. Each task is scheduled on a single physical machine. When a job is submitted, the user can specify the maximum allowed resource demand for each task in terms of required CPU and memory size. Dynamically adjust the number of active machines in a data center in order to reduce energy consumption while meeting the service level objectives (SLOs) of workloads. The coordinates of each point in these figures correspond to a combination of CPU and memory requirements.

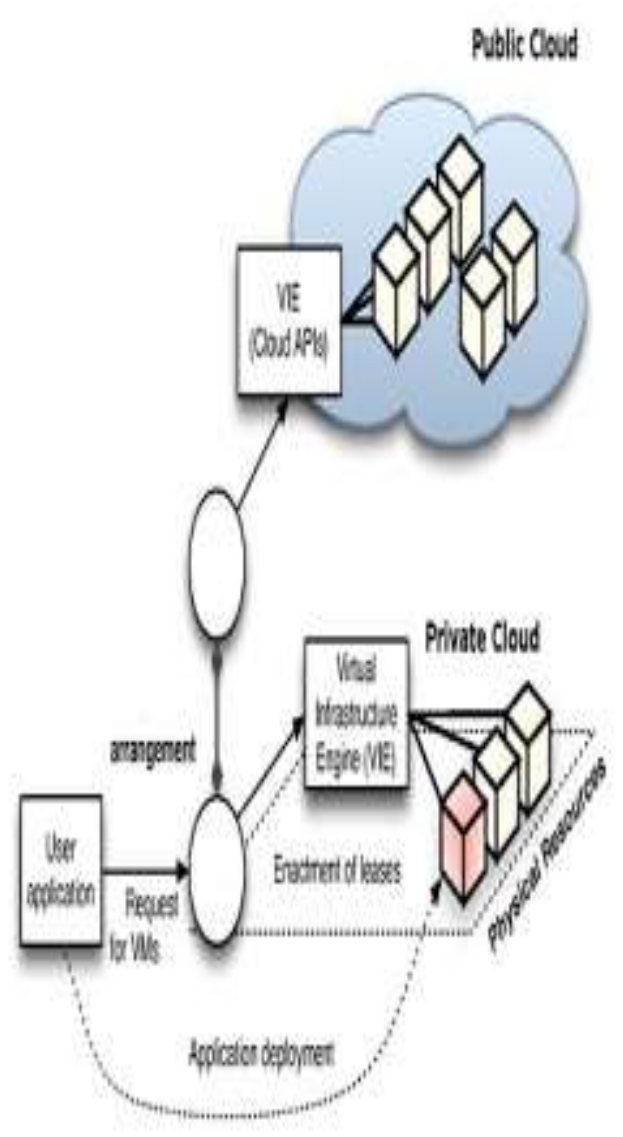

Figure 1. Architecture of proposed framework

The resource provisioning in Cloud Computing, the long-held dream of computing as a utility, has the potential to transform a large part of the IT industry, making software even more attractive as a service and shaping the way IT hardware is designed and purchased. Developers with innovative ideas for new Internet services no longer require the large capital outlays in hardware to deploy their service or the human expense to operate it. They need not be concerned about overprovisioning for a service whose popularity does not meet their predictions, thus wasting costly resources, or underprovisioning for one that becomes wildly popular, thus missing potential customers and revenue. The Methodology based on Infrastructure as a Service layer to access resources on-demand. A Rule Based Resource Manager is proposed to scale up private cloud and presents a cost effective solution in terms of money spent to scale up private cloud on-demand by taking public cloud's resources and that never permits secure information to cross the organization's firewall in hybrid cloud. Also set the time for public cloud and private cloud to fulfill the request.

\section{CONCLUSION}

The user's usages have large number of progress in an environment. So there have large number of problems are occurred in the cloud. The resource provisioning can be overcome by hybrid method. This proposed method is used to allocate the resources with working conditions. It shows the energy is very efficiency and the overcome the workload with the good performance. 


\section{REFERENCES}

[1]http://www.youtube.com/yt/press/statistics.html [2] http://nlp.stanford.edu/software/corenlp.shtml [3]Collins English Dictionary, entry for "lemmatise" [4]L. Ratinov and D. Roth, Design Challenges and Misconceptions in Named Entity Recognition. CoNLL (2009) [5]G. A. Miller.Wordnet: A lexical database for english. (11):39-41.

[6]Chengde Zhang, Xiao Wu, Mei-Ling Shyu and QiangPeng, " Adaptive Association Rule Mining for Web Video Event Classification", 2013 IEEE 14th International Conference on Information Reuse and Integration (IRI), page 618-625.

[7] Y. Song, M. Zhao, J. Yagnik, and X. Wu.Taxonomic classification for web-based videos.In CVPR, 2010.

[8] Z. Wang, M. Zhao, Y. Song, S. Kumar, and B. Li. Youtube-cat: Learning to categorize wild web videos. In CVPR, 2010.

[9] http://www.ranks.nl/resources/stopwords.html [10] http://cs.nyu.edu/grishman/jet/guide/PennPOS.html [11]Roth and D. Zelenko, Part of Speech Tagging Using a Network of Linear Separators. Coling-Acl, The 17th International Conference on Computational Linguistics (1998) pp. $1136-1142$
[12]O. Duchenne, I. Laptev, J. Sivic, F. Bach, and J. Ponce. $\mathrm{Au}$ - tomatic annotation of human actions in video. In Proc. of ICCV, 2009.

[13]Laptev, M. Marszalek, C. Schmid, and B. Rozenfeld.Learning realistic human actions from movies. In Proc. of CVPR, 2008

[14]M. Everingham, J. Sivic, and A. Zisserman. Hello! my name is... buffy automatic naming of characters in tv video. In Proc. of BMVC, 2006.

[15]F. Smeaton, P. Over, and W. Kraaij.Evaluation campaigns and trecvid. In Proc. of ACM Workshop on Multimedia Information Retrieval, 2006

[16]J. Yang, R. Yan, and A. G. Hauptmann. Cross-domain video concept detection using adaptive svms. In Proc. of ACM MM, 2007.

[17] M. E. Sargin, H. Aradhye, P. J. Moreno, and M. Zhao. $\mathrm{Au}$ - diovisual celebrity recognition in unconstrained web videos. In Proc. of ICASSP, 2009.

[18] J. Liu, J. Luo, and M. Shah.Recognizing realistic actions from videos.In Proc. of CVPR, 2009.

[19] S. Zhang, C. Zhu, J. K. O. Sin, and P. K. T. Mok, "A novel ultrathin elevated channel low-temperature poly-Si

TFT," IEEE Electron Device Lett., vol. 20, pp. 569-571, Nov. 1999 